\title{
Neural network study of the nucleon axial form-factor
}

\author{
Luis Alvarez-Ruso \\ Instituto de Física Corpuscular (IFIC) and Departamento de Física Teórica, Centro Mixto \\ UVEG-CSIC, Valencia, Spain \\ E-mail: Luis.Alvarez@ific.uv.es \\ Krzysztof M. Graczyk \\ Institute of Theoretical Physics, University of Wrocław, pl. M. Borna 9, 50-204, Wrocław, Poland \\ E-mail: Krzysztof.Graczykeift.uni.wroc.pl
}

\section{Eduardo Saul-Sala*t}

Departamento de Física Teórica and Instituto de Física Corpuscular (IFIC), Centro Mixto UVEG-CSIC, Valencia, Spain

E-mail: Eduardo.Saul@ific.uv.es

\begin{abstract}
We have performed the first Bayesian neural-network analysis of neutrino-deuteron scattering data [1]. The nucleon axial form factor has been extracted from quasielastic scattering data collected by the Argonne National Laboratory (ANL) bubble chamber experiment using a modelindependent parametrization. The results are in agreement with previous determinations only when the low $0.05<Q^{2}<0.10 \mathrm{GeV}^{2}$ region is excluded from the analysis. This suggests that corrections from the deuteron structure may play a crucial role at low $Q^{2}$, although experimental errors in this kinematic region could have also been underestimated. With new and more precise measurements of neutrino-induced quasielastic scattering on hydrogen and deuterium, the present framework would be readily applicable to unravel the axial structure of the nucleon.
\end{abstract}

The 20th International Workshop on Neutrinos (NuFact2018)

12-18 August 2018

Blacksburg, Virginia

\footnotetext{
${ }^{*}$ Speaker.

$\dagger$ The calculations have been carried out in the Wroclaw Centre for Networking and Supercomputing (http://www.wcss.wroc.pl), grant No. 268. This work has been partially supported by the Spanish Ministerio de Economía y Competitividad and the European Regional Development Fund, under contracts FIS2014-51948-C2-1-P, FIS2014-51948-C2-2-P, FIS2017-84038-C2-1-P, FIS2017-84038-C2-1-P and SEV-2014-0398 and by Generalitat Valenciana under contract PROMETEOII/2014/0068.
} 


\section{Introduction}

A deep understanding of neutrino interactions with matter is crucial for oscillations experiments aiming at the determination of neutrino properties. To reach the goals of precision and accuracy desired, a realistic modeling of neutrino interactions with nuclei is required. The nucleon axial form factor, $F_{A}$, is a source of uncertainty in the amplitudes and therefore in the cross sections, which are the key ingredient of the interaction models. The axial form factor is a fundamental property of the nucleon and is a function of the momentum transferred in the interaction, $-Q^{2}$. The most common parametrization used in the literature is the dipole ansatz which depends only on a single parameter, $M_{A}$, and is not theoretically well founded.

Bubble chamber experiments of neutrino scattering on deuterium collected a data set for the quasielastic process, from which $F_{A}$ can be extracted. For this purpose we have performed a semiparametric analysis and obtained model-independent information about $F_{A}$ from the ANL experimental data $[2,3,4]$. For the analysis we have used feed-forward neural networks in a multilayer perceptron (MLP) configuration [5]. The tool that allows us to choose between all the different results given by the neural networks is Bayesian statistics [6]. This framework has been applied to the extraction of electromagnetic form factor from electron scattering data [7].

\section{Numerical results and summary}

It is known that the low- $Q^{2}$ data are characterized by a lower efficiency (see for instance Fig. 1 of ref. [4]). Moreover, in this kinematic domain deuteron structure corrections must be carefully taken into account. In order to study this problem we have considered three variants of the ANL data: including all the bins (BIN0) and without the first $k$ bins (BIN $k$ ), where $k=1$ or $k=2$. Additionally, for each data set we considered the cross section model with and without deuteron corrections, for which we use the calculation of Ref. [8] (solid line of Fig. 4 in this reference).

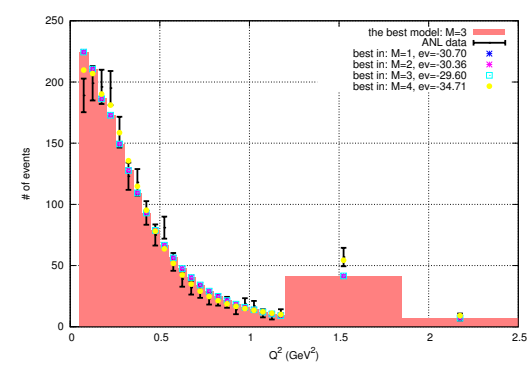

Figure 1: Best fits obtained for MLPs with $M=1-4$ hidden units in the analysis of the BIN1 data set with deuteron corrections (log of evidence given). Black bars denote the ANL number of events in each $Q^{2}$ bin.

We adopted MLP configurations with 1-4 hidden units in a single hidden layer. As can be seen in Fig. 1, all the best models within each MLP type reproduce well the ANL data. The fits including all bins, Fig. 2, show a positive slope at low $Q^{2}$ which result in a negative value for the axial radius squared, $r_{A}^{2}$, incompatible with all the available determinations. The height of the $F_{A}$ local maximum is reduced once the deuteron correction is included; this maximum disappears when the first bin has been removed from the ANL data. The value of $r_{A}$ for the best fit corresponds to the BIN2 data and has a value compatible with other determinations such as the z-expansion ones from bubble chamber data [9] and from muon capture by protons [10] but with a significantly smaller error [1]. We realize that deuteron effects are sizable by looking at the changes in the behavior of 

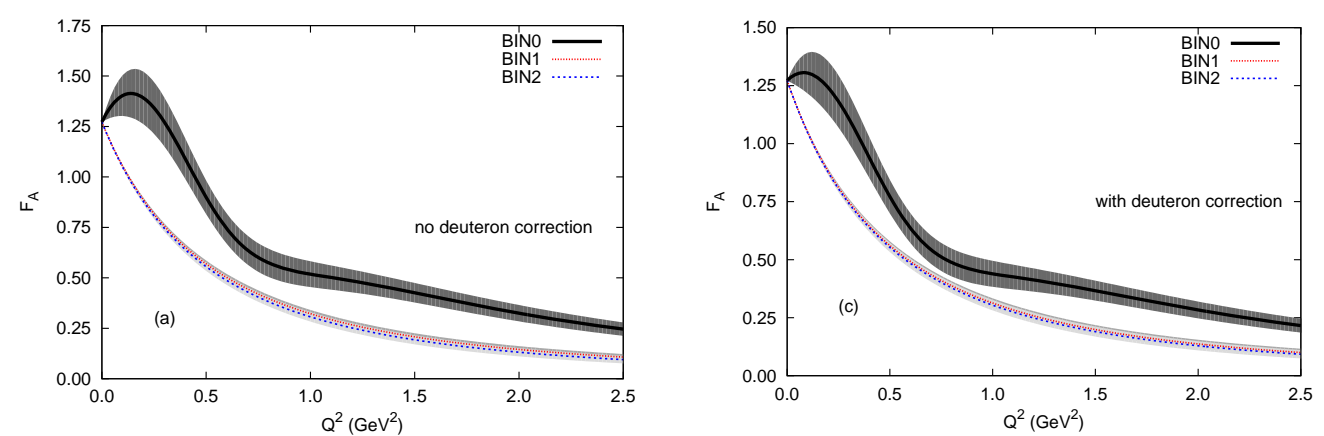

Figure 2: Left (right) panel shows the best fit to the the BIN0, BIN1 and BIN2 data sets, obtained without (with) deuteron corrections. The shaded areas denote $1 \sigma$ uncertainties of $F_{A}$.

$F_{A}$ at low $Q^{2}$ when we add the deuteron corrections and when we take out the first bins of the data. At low $Q^{2}$, a poorly understood efficiency could also have an impact on the results. In the case of BIN1 and BIN2 sets, the present analysis does not show significant deviations from the dipole ansatz. On the other hand, Bayesian techniques allow us to avoid both over-fitting and under-fitting, thus neural-network fits have smaller uncertainties compared to dipole or other determinations.

With new more precise data from (anti)neutrino scattering on hydrogen and/or deuterium (see Ref. [11] for a novel proposal), techniques like the one applied in the present study shall prove valuable to unravel the axial structure of the nucleon.

\section{References}

[1] L. Alvarez-Ruso, K. M. Graczyk and E. Saul-Sala, Nucleon axial form factor from a Bayesian neural-network analysis of neutrino-scattering data, Phys. Rev. C99 (2019) 025204 [1805. 00905 ].

[2] W. A. Mann et al., Study of the reaction $v n \rightarrow \mu^{-}$, Phys. Rev. Lett. 31 (1973) 844.

[3] S. J. Barish et al., Study of Neutrino Interactions in Hydrogen and Deuterium. 1. Description of the Experiment and Study of the Reaction $v d \rightarrow \mu^{-} p p_{s}$, Phys. Rev. D16 (1977) 3103.

[4] K. L. Miller et al., STUDY OF THE REACTION $v_{\mu} d \rightarrow \mu^{-}$pp $p_{s}$, Phys. Rev. D26 (1982) 537.

[5] K. Hornik, M. Sinchcombe and W. Halbert, Multilayer feedforward networks are universal approximators, Neural Networks 2 (1989) 359.

[6] G. D’Agostini, Bayesian Reasoning in Data Analysis. World Scientific, 2003.

[7] K. M. Graczyk, P. Plonski and R. Sulej, Neural Network Parameterizations of Electromagnetic Nucleon Form Factors, JHEP 09 (2010) 053 [1 006 . 0342].

[8] S. K. Singh and H. Arenhovel, Pion exchange current effects in $v_{\mu}+d \rightarrow \mu^{-}+p+p$, Z. Phys. A324 (1986) 347.

[9] A. S. Meyer, M. Betancourt, R. Gran and R. J. Hill, Deuterium target data for precision neutrino-nucleus cross sections, Phys. Rev. D93 (2016) 113015 [1603. 03048 ].

[10] R. J. Hill, P. Kammel, W. J. Marciano and A. Sirlin, Nucleon Axial Radius and Muonic Hydrogen - A New Analysis and Review, Rept. Prog. Phys. 81 (2018) 096301 [1708.08462].

[11] H. Duyang, B. Guo, S. R. Mishra and R. Petti, A Novel Approach to Neutrino-Hydrogen Measurements, 1809.08752. 